\title{
Post-surgical Infective Endocarditis Involving Ghordae Tendinae: A Rare Presentation
}

Shankar Kalgudi, Pradeep Rangappa, Prabhakar Shetty', Karthik Rao

From the Intensive Care Unit and Department of Cardiology', Columbia

Asia Referral Hospital, Yeshwantpur, Bangalore - 560055, India.

\begin{abstract}
:
Transient bacteraemia following surgical procedures is a relatively common, but concomitant infective endocarditis in patient without any risk factor is a rare event. To the best of our knowledge, this is one of the few case reports of infective endocarditis following a urology procedure. We report a 57-year-old lady with pyelonephritis and renal calculus who developed vegetation's on chordae tendinae following renal surgery.
\end{abstract}

Key words: Endocarditis, Pyelonephritis, Kidney Calculi, Chordae tendinae, Bacteremia.

\section{Introduction}

Infective endocarditis (IE) is an infection of the endocardial surface of the heart. The intracardiac effects of this infection include severe valvular insufficiency, which may lead to intractable congestive heart failure and myocardial abscesses [1]. IE also produces a wide variety of systemic signs and symptoms through several mechanisms, including both sterile and infected emboli and various immunological phenomena [1].

Cases of nosocomial infective endocarditis (NIE), intravenous drug abuse (IVDA) IE, and prosthetic valve endocarditis (PVE) are quite common [2]. Other rare variants of IE are post-surgical and blood culture negative infective endocarditis ( $B C N$ IE). Literature search has yielded very few case reports following surgery and with contributing risk factors [3]. BCN-IE is a rare variant and is defined as endocarditis without etiology and blood cultures yield no growth of organisms. BCN-IE with vegetations arising from chordae tendinae is not previously reported.

\section{Case Report}

A 57 year old lady was admitted to the ICU as first admission with 4 days history of fever, chills and rigors, burning micturition, diffuse abdominal pain, shortness of breath and bilateral pedal edema since last 10 days. She had a past medical history of diabetes mellitus, hypertension and hypothyroidism.

She was diagnosed to have acute pyelonephritis with swollen right kidney and right renal calculus of $4 \mathrm{~mm}$. The patient was resuscitated with fluids boluses, started on antibiotics imipenem - cilastatin

\section{Corresponding Author: Dr. Pradeep Rangappa}

Email: drpradeepr@aol.com

Received: March 4, 2014 | Accepted: March 24, 2014 | Published Online: April 10, 2014

This is an Open Access article distributed under the terms of the Creative Commons Attribution License (creativecommons.org/licenses/by/3.0)

Conflict of interest: None declared | Source of funding: Nil | DOl: http://dx.doi.org/10.17659/01.2014.0029 
and underwent right ureteroscopy with DJ stent placement with drainage of frank pus. Preoperative echocardiography revealed normal LV function with no vegetations. Post procedure her renal function tests and urine output improved. She was discharged home on tablet nitrofurantoin for 7 days and advised right retrograde intrarenal (RIRS) surgery after 2 weeks for removal of calculi

After 2 weeks, she was readmitted and underwent RIRS procedure. Post RIRS, she developed severe pain abdomen in left hypochondriac region, tachycardia with hypotension requiring intubation with mechanical ventilation and vasopressor supports. Computed tomography of abdomen revealed multiple splenic infarcts, enlarged lymph nodes and bilateral pulmonary consolidation in lungs. Echo revealed a normal LV function, a rounded opacity measuring $1.6 \times 0.8 \mathrm{~cm}$ attached to chordae tendinae extending to anterior mitral leaflets (AML) [Fig.1], mild to moderate $M R$, dilated right sided chambers, moderate tricuspid regurgitation and pulmonary artery pressures of

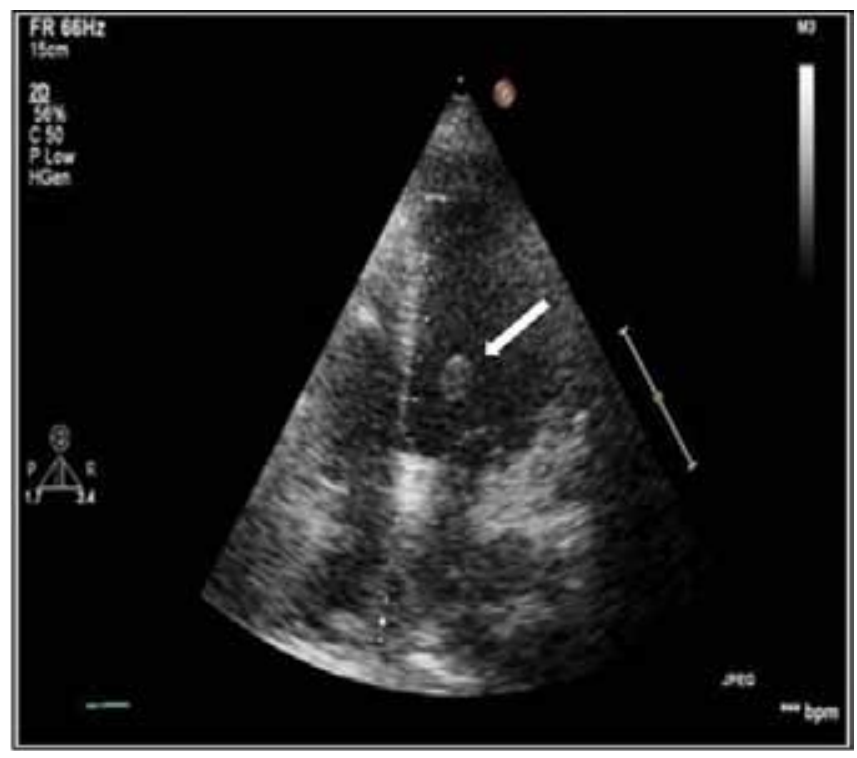

Fig.1: Transthoracic echocardiography (TTE) showing vegetations arising from chordae tendinae.
$60 \mathrm{~mm} \mathrm{Hg}$. Three sets of blood culture showed no growth. The patient fulfilled Dukes 1 major criterion, viz, evidence of vegetations confirmed by echocardiogram and 2 minor criteria, viz, splenic infarcts and fever.

She was empirically started on antibiotics ertapenem and teicoplanin. Diagnosis of post surgical IE with vegetations arising from chordae tendinae was made. Trans-oesophageal echocardiogram (TEE) confirmed the presence of vegetations on chordae tendinae. By post operative day 10, she had stabilized and was discharged home on piperacillin -tazobactam and vancomycin, with advice for review after 2 weeks. She remained comfortable and hemodynamically stable during the follow ups. After completing the full course of 6 weeks of antibiotics, echo revealed complete resolution of vegetations [Fig.2] with normal LV function. Blood culture sent during each follow up showed no growth of organisms.

Our case report is unique and rare as in IE had developed following surgical procedure with no

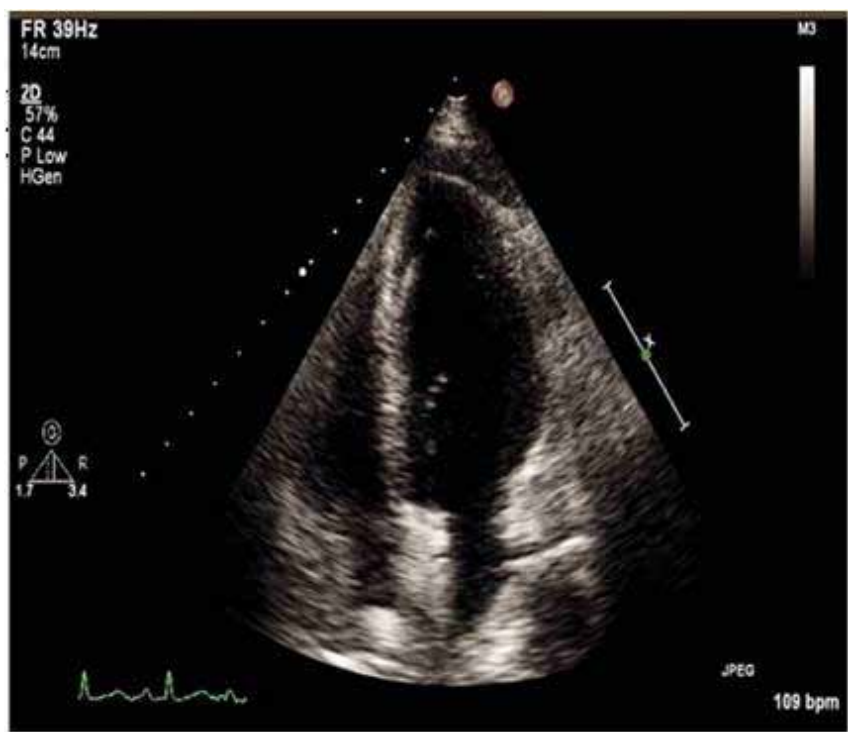

Fig.2: TTE after six weeks of antibiotics showing resolution of vegetations. 
risk factors, blood cultures showed no growth of organisms and there was a presentation of vegetations arising from chordae tendinae.

\section{Discussion}

IE is a systemic disease which develops in patients with underlying congenital heart disease, cardiac valvulopathy and presents with various clinical symptoms of infection, embolization and cardiac symptoms [1]. Initially IE occurs by formation of a sterile vegetations (contains platelets, fibrin and blood clots) on a valve that is damaged by valvular disease [4]. Vegetations usually occur at a site associated with high-speed blood flow or direct collision with the endocardial surface like valves (native or prosthetic), interventricular septum, mural endocardium and intracardiac devices. Onset is rare at a site where normal-speed blood flow exists [4]. Sterile vegetations may get infected during/following transient bacteraemia associated with surgical procedures in the fields of dentistry, otorhinolaryngology, urology, and obstetrics and gynaecology [5]. However, the frequency is very low as most patients at risk receive prophylactic antibiotics. Also, typical physical findings such as a fever and generalised fatigability are inconsistent, hence it is detected late [4]. Literature search has yielded only one case report following urology procedure wherein the patient had pre-existing mitral valve disease as a risk factor [3]. Our patient had no contributing risk factors and IE occurred following urology procedure.

Causative bacteria have been identified by blood culture in 70 to $80 \%$ of all patients [2]. Amongst causative bacteria, aerobic gram-positive and anaerobic bacteria account for 70 to $80 \%$, including Staphylococcus aureus, Streptococcus viridans, Enterococcus, coagulase - negative Staphylococcus, etc $[6,7]$. In the present case, blood cultures were performed 4 times but no organism was isolated. Hence, it was diagnosed as culture negative endocarditis which is seen in 2.5 to $31 \%$ of patients [8]. BCN-IE is an infection with inflammation of the lining of one or more heart valves in which no endocarditis - causing germs can be identified on a blood culture. The reasons for this are that certain germs do not grow well in the laboratory setting, or that some patients may have previously received antibiotics that keep such germs from growing [8]. Other causes include right sided endocarditis, prosthetic valve endocarditis, collagen vascular diseases and because of organisms which are slow growing or fastidious in nature [8].

Literature search showed vegetations mainly arising from valves (native or prosthetic), interventricular septum, mural endocardium and intracardiac devices [4]. In our patient, vegetations originated from chordae tendinae and our differential diagnosis was fibroelastoma of chordae tendinae, chordae tendinae rupture, vegetations arising from valves and extending to chordae tendinae and post mitral valve repair with retained chordae tendinae. Trans-esophageal echocardiography done however ruled out the above differentials.

\section{Conclusion}

Early recognition of IE in post surgical setting and awareness of its origin from unusual sites with appropriate treatment results in good patient recovery.

\section{References}

1. Braunwald E, Zipes DP, Libby $P$, Bonow R. Braunwald's Heart disease: A text book of cardiovascular medicine. $9^{\text {th }}$ ed. Elsevier Saunders; 2011.

2. Fauci AS, Braunwald E, Kasper DL, Hauser SL, Longo DL, Jameson JL, Loscalzo J. Harrison's Principles of Internal Medicine. $17^{\text {th }}$ ed. New York: McGraw-Hill; 2008.

3. Kawahara T, Taguchi H, Yamagishi T, Udagawa 
K, Ouchi H, Misaki H. A case of infective endocarditis after transurethral prostate resection. Urology annals. 2010;2(2):83-85

4. Churchill MA Jr, Geraci JE, Hunder GG. Musculoskeletal manifestations of bacterial endocarditis . Ann Intern Med. 1977;87(6):754759.

5. Woo PC, Fung AM, Lau SK, Chan BY, Chiu SK, Teng JL, et al. Granulicatella adiacens and abiotrophia defective bacteraemia characterized by $16 \mathrm{~S}$ r RNA gene sequencing. J
Med Microbiol. 2003;52(Pt 2):137-140.

6. Wermer AS, Cobbs CG, Kaye D, Hook EW. Studies on the bacteraemia of bacterial endocarditis. JAMA.1967;202(3):199-203.

7. Peterson CE, Cook JL, Burke JP. Mediadependant subculture of nutritionally variant streptococci. Am J Clin Pathol. 1981 ;75(4):634636.

8. Werner M, Andersson R, Olaison L, Hogevik HA. Clinical study of culture-negative endocarditis. Medicine (Baltimore). 2003;82(4):263-273. 